\author{
LINEAR AND NON-LINEAR THEORY OF \\ GENERALIZED FUNCTIONS AND ITS APPLICATIONS \\ BANACH CENTER PUBLICATIONS, VOLUME 88 \\ INSTITUTE OF MATHEMATICS \\ POLISH ACADEMY OF SCIENCES \\ WARSZAWA 2010
}

\title{
KERNEL THEOREMS IN SPACES OF GENERALIZED FUNCTIONS
}

\author{
ANTOINE DELCROIX \\ Equipe Analyse Algébrique Non Linéaire \\ Laboratoire Analyse, Optimisation, Contrôle \\ Faculté des sciences - Université des Antilles et de la Guyane \\ 97159 Pointe-à-Pitre Cedex, Guadeloupe (France) \\ E-mail: Antoine.Delcroix@univ-ag.fr
}

\begin{abstract}
In analogy to the classical isomorphism between $\mathcal{L}\left(\mathcal{D}\left(\mathbb{R}^{n}\right), \mathcal{D}^{\prime}\left(\mathbb{R}^{m}\right)\right)$ and $\mathcal{D}^{\prime}\left(\mathbb{R}^{m+n}\right)$ (resp. $\mathcal{L}\left(\mathcal{S}\left(\mathbb{R}^{n}\right), \mathcal{S}^{\prime}\left(\mathbb{R}^{m}\right)\right)$ and $\left.\mathcal{S}^{\prime}\left(\mathbb{R}^{m+n}\right)\right)$, we show that a large class of moderate linear mappings acting between the space $\mathcal{G}_{C}\left(\mathbb{R}^{n}\right)$ of compactly supported generalized functions and $\mathcal{G}\left(\mathbb{R}^{n}\right)$ of generalized functions (resp. the space $\mathcal{G}_{\mathcal{S}}\left(\mathbb{R}^{n}\right.$ ) of Colombeau rapidly decreasing generalized functions and the space $\mathcal{G}_{\tau}\left(\mathbb{R}^{n}\right)$ of temperate ones) admits generalized integral representations, with kernels belonging to specific regular subspaces of $\mathcal{G}\left(\mathbb{R}^{m+n}\right)$ (resp. $\mathcal{G}_{\tau}\left(\mathbb{R}^{m+n}\right)$ ). The main novelty is to use accelerated $\delta$-nets, which are unit elements for the convolution product in these regular subspaces, to construct the kernels. Finally, we establish a strong relationship between these results and the classical ones.
\end{abstract}

1. Introduction. During the last three decades, theories of nonlinear generalized functions have been developed by many authors (see 1, 11, 13, 14 and others), mainly based on the ideas of J.-F. Colombeau [3, 4, which we are going to follow in the sequel. These theories appear to be a natural continuation of distribution theory [12, 19, 20, specially efficient to pose and solve differential or integral problems with irregular data.

We continue the investigations, initiated by [16] (published in [17, 18]) and carried on by [2, 15, 7, 8, 10, 21], in the field of generalized integral operators (which generalize in the Colombeau framework the operators with distributional kernels). More precisely, we improve and give the final version of Schwartz kernel type theorems published in [5], thanks to a remark by Dimitris Scarpalézos (private communication): In some specific subspaces

2000 Mathematics Subject Classification: Primary 47G10; Secondary 45P05, 46F05, 46F30.

Key words and phrases: kernel theorems, nuclear spaces, Colombeau generalized functions, Colombeau temperate generalized functions, integral operator, Schwartz distributions, tempered distributions.

The paper is in final form and no version of it will be published elsewhere. 
of Colombeau type algebras, accelerated $\delta$-nets are unit elements for the product of convolution. This property makes it possible to remove some technical assumptions. Thus, we can give completely similar results as the nuclear type theorems of [7] and compare all these results with the one of [21.

Our starting point is a moderate net of linear maps $\left(L_{\varepsilon}: \mathcal{D}\left(\mathbb{R}^{n}\right) \rightarrow \mathrm{C}^{\infty}\left(\mathbb{R}^{m}\right)\right)_{\varepsilon}$ (resp. $\left.\left(L_{\varepsilon}: \mathcal{S}\left(\mathbb{R}^{n}\right) \rightarrow \mathcal{O}_{M}\left(\mathbb{R}^{m}\right)\right)_{\varepsilon}\right)$, that is, satisfying some growth properties with respect to the parameter $\varepsilon$, which gives rise to a linear map $L: \mathcal{G}_{C}\left(\mathbb{R}^{n}\right) \rightarrow \mathcal{G}\left(\mathbb{R}^{m}\right.$ ) (resp. $\left.L: \mathcal{G}_{S}\left(\mathbb{R}^{n}\right) \rightarrow \mathcal{G}_{\tau}\left(\mathbb{R}^{n}\right)\right)$. The symbols $\mathcal{G}\left(\mathbb{R}^{m}\right), \mathcal{G}_{C}\left(\mathbb{R}^{n}\right), \mathcal{G}_{S}\left(\mathbb{R}^{n}\right)$ and $\mathcal{G}_{\tau}\left(\mathbb{R}^{m}\right)$ denote respectively the space of generalized functions, of compactly supported ones, of rapidly decreasing and of tempered ones. These spaces play respectively the role of $\mathrm{C}^{\infty}\left(\mathbb{R}^{m}\right)$, $\mathcal{D}\left(\mathbb{R}^{n}\right), \mathcal{S}\left(\mathbb{R}^{n}\right)$ and $\mathcal{S}^{\prime}\left(\mathbb{R}^{m}\right)$ in the corresponding classical kernel theorems. The main result (Theorems 3.6 and 3.7) is that $L$ can be represented as a generalized integral operator in the spirit of Schwartz Kernel Theorem (resp. kernel theorem for nuclear spaces). The use of accelerated $\delta$-nets is a fundamental tool to obtain results valid with no restriction in spaces of generalized functions with sublinear asymptotic growth. Note that we correct here the definitions given in [7] for the case of regular subspaces of $\mathcal{G}_{\tau}\left(\mathbb{R}^{m}\right)$. Furthermore, these results contain the classical isomorphism theorem recalled above in the following sense. We can associate to each linear continuous operator $\Lambda: \mathcal{D}\left(\mathbb{R}^{n}\right) \rightarrow \mathcal{D}^{\prime}\left(\mathbb{R}^{m}\right)$ (resp. $\left.\mathcal{S}\left(\mathbb{R}^{n}\right) \rightarrow \mathcal{S}^{\prime}\left(\mathbb{R}^{m}\right)\right)$ a moderate map $L_{\Lambda}: \mathcal{G}_{C}\left(\mathbb{R}^{n}\right) \rightarrow \mathcal{G}\left(\mathbb{R}^{m}\right)\left(\operatorname{resp} . L_{\Lambda}: \mathcal{G}_{S}\left(\mathbb{R}^{n}\right) \rightarrow \mathcal{G}_{\tau}\left(\mathbb{R}^{n}\right)\right)$ and consequently a kernel $H_{L_{\Lambda}} \in \mathcal{G}\left(\mathbb{R}^{m+n}\right)\left(\right.$ resp. $\left.\mathcal{G}_{\tau}\left(\mathbb{R}^{m+n}\right)\right)$ such that, for all $f$ in $\mathcal{D}\left(\mathbb{R}^{n}\right)$ $\left(\right.$ resp. $\left.\mathcal{S}\left(\mathbb{R}^{n}\right)\right), \Lambda(f)$ and $\widetilde{H}_{L_{\Lambda}}(f)$ are equal, where $\widetilde{H}_{L_{\Lambda}}$ is the integral operator associated with $H_{L_{\Lambda}}$ (Proposition 3.9).

The next steps in this topic would be to introduce a complete theory of nuclear spaces in the framework of Colombeau algebras and to establish Kernel Theorems directly for linear mappings acting on $\widetilde{\mathbb{C}}$ modules, following [9] and further works in the same direction.

2. Colombeau type algebras. Throughout this section $d$ will be a strictly positive integer and $\Omega$ an open subset of $\mathbb{R}^{d}$. For $f \in \mathrm{C}^{\infty}(\Omega), r \in \mathbb{Z}, l \in \mathbb{N}$ and $K \subset \subset \Omega$ set

$$
p_{K, l}(f)=\sup _{x \in K,|\alpha| \leq l}\left|\partial^{\alpha} f(x)\right|, \quad \mu_{r, l}(f)=\sup _{x \in \Omega,|\alpha| \leq l}(1+|x|)^{r}\left|\partial^{\alpha} f(x)\right|
$$

with values in $[0,+\infty]$ for the latter.

\subsection{Sheaves and presheaves of regular generalized functions}

Definition $2.1([6])$. A subspace $\mathcal{R}$ of $\mathbb{R}_{+}^{\mathbb{N}^{i}}(i=1,2)$ is regular if it is nonempty and:

(i) $\mathcal{R}$ is "overstable" by translation and by maximum, that is

$$
\begin{gathered}
\forall N \in \mathcal{R}, \quad \forall a \in \mathbb{N}^{i}, \quad \forall b \in \mathbb{R}_{+}, \exists N^{\prime} \in \mathcal{R}, \quad N(\cdot+a)+b \leq N^{\prime}(\cdot), \\
\forall N_{1} \in \mathcal{R}, \quad \forall N_{2} \in \mathcal{R}, \quad \exists N \in \mathcal{R}, \quad \max \left(N_{1}, N_{2}\right) \leq N .
\end{gathered}
$$

(ii) For all $N_{1}$ and $N_{2}$ in $\mathcal{R}$, there exists $N \in \mathcal{R}$ such that

$$
\forall a, b \in \mathbb{N}^{i}, \quad N_{1}(a)+N_{2}(b) \leq N(a+b) .
$$

EXAmple 2.2. The set $\mathcal{B}$ of bounded sequences of $\mathbb{R}_{+}^{\mathbb{N}}$ is a regular subset of $\mathbb{R}_{+}^{\mathbb{N}}$, which is itself regular. 
In the sequel " $\mathrm{O}\left(\varepsilon^{p}\right)$ as $\varepsilon \rightarrow 0$ " is abbreviated to " $\mathrm{O}\left(\varepsilon^{p}\right)$ ". Take $\mathcal{R}$ and $\Lambda$ two regular subsets of $\mathbb{R}_{+}^{\mathbb{N}}$ and $\mathcal{R}_{2}$ a regular subset of $\mathbb{R}_{+}^{\mathbb{N}^{2}}$. Set

$$
\begin{aligned}
\mathcal{E}_{M}^{\mathcal{R}}(\Omega) & =\left\{\left(f_{\varepsilon}\right)_{\varepsilon} \in \mathrm{C}^{\infty}(\Omega)^{(0,1]} \mid \forall K \subset \subset \Omega, \exists N \in \mathcal{R}, \forall l \in \mathbb{N}, p_{K, l}\left(f_{\varepsilon}\right)=\mathrm{O}\left(\varepsilon^{-N(l)}\right)\right\}, \\
\mathcal{E}_{\tau}^{\mathcal{R}, \Lambda}(\Omega) & =\left\{\left(f_{\varepsilon}\right) \in \mathcal{O}_{M}(\Omega)^{(0,1]} \mid \exists(N, \lambda) \in \mathcal{R} \times \Lambda, \forall l \in \mathbb{N}, \mu_{-\lambda(l), l}\left(f_{\varepsilon}\right)=\mathrm{O}\left(\varepsilon^{-N(l)}\right)\right\}, \\
\mathcal{E}_{\mathcal{S}}^{\mathcal{R}_{2}}(\Omega) & =\left\{\left(f_{\varepsilon}\right)_{\varepsilon} \in \mathcal{S}(\Omega)^{(0,1]} \mid \exists N \in \mathcal{R}_{2}, \forall(q, l) \in \mathbb{N}^{2}, \quad \mu_{q, l}\left(f_{\varepsilon}\right)=\mathrm{O}\left(\varepsilon^{-N(q, l)}\right)\right\} .
\end{aligned}
$$

Note that, in the definition of $\mathcal{E}_{\tau}^{\mathcal{R}, \Lambda}(\Omega)$, two regular subsets are involved. One is linked with the growth of $\left(f_{\varepsilon}\right)_{\varepsilon}$ with respect to $\varepsilon$ and the other to the growth with respect to $1+|x|$. Set

$$
\begin{aligned}
\mathcal{N}(\Omega) & =\left\{\left(f_{\varepsilon}\right)_{\varepsilon} \in \mathrm{C}^{\infty}(\Omega)^{(0,1]} \mid \forall K \subset \subset \Omega, \forall l \in \mathbb{N}, \forall m \in \mathbb{N}, \quad p_{K, l}\left(f_{\varepsilon}\right)=\mathrm{O}\left(\varepsilon^{m}\right)\right\}, \\
\mathcal{N}_{\tau}(\Omega) & =\left\{\left(f_{\varepsilon}\right)_{\varepsilon} \in \mathcal{O}_{M}(\Omega)^{(0,1]} \mid \forall l \in \mathbb{N}, \exists q \in \mathbb{N}, \forall m \in \mathbb{N}, \quad \mu_{-q, l}\left(f_{\varepsilon}\right)=\mathrm{O}\left(\varepsilon^{m}\right)\right\}, \\
\mathcal{N}_{\mathcal{S}}(\Omega) & =\left\{\left(f_{\varepsilon}\right)_{\varepsilon} \in \mathcal{S}(\Omega)^{(0,1]} \mid \forall(q, l) \in \mathbb{N}^{2}, \forall m \in \mathbb{N}, \quad \mu_{q, l}\left(f_{\varepsilon}\right)=\mathrm{O}\left(\varepsilon^{m}\right)\right\} .
\end{aligned}
$$

Finaly, set

$$
\begin{aligned}
\mathcal{E}_{M}(\mathbb{C}) & =\left\{\left(r_{\varepsilon}\right)_{\varepsilon} \in \mathbb{C}^{(0,1]}|\exists N \in \mathbb{N},| r_{\varepsilon} \mid=\mathrm{O}\left(\varepsilon^{-N}\right)\right\}, \\
\mathcal{N}(\mathbb{K}) & =\left\{\left(r_{\varepsilon}\right)_{\varepsilon} \in \mathbb{C}^{(0,1]}|\forall m \in \mathbb{N},| r_{\varepsilon} \mid=\mathrm{O}\left(\varepsilon^{m}\right)\right\} .
\end{aligned}
$$

The functor $\mathcal{E}_{M}^{\mathcal{R}}(\cdot)$ defines a sheaf of differential algebras over the ring $\mathcal{E}_{M}(\mathbb{C})$ whereas the functor $\mathcal{E}_{\tau}^{\mathcal{R}, \Lambda}(\cdot)$ (resp. $\mathcal{E}_{\mathcal{S}}^{\mathcal{R}_{2}}(\cdot)$ ) defines a presheaf of differential algebras over $\mathcal{E}_{M}(\mathbb{C})$. The functor $\mathcal{N}(\cdot)$ (resp. $\mathcal{N}_{\tau}(\cdot), \mathcal{N}_{\mathcal{S}}(\cdot)$ ) defines a sheaf (resp. presheaves) of ideals of the sheaf $\mathcal{E}_{M}^{\mathcal{R}}(\cdot)$ (resp. $\left.\mathcal{E}_{\tau}^{\mathcal{R}, \Lambda}(\cdot), \mathcal{E}_{\mathcal{S}}^{\mathcal{R}_{2}}(\cdot)\right)$. Moreover, $\mathcal{G}^{\mathcal{R}}(\cdot)=\mathcal{E}_{M}^{\mathcal{R}}(\cdot) / \mathcal{N}(\cdot)$ (resp. $\mathcal{G}_{\tau}^{\mathcal{R}, \Lambda}(\cdot)=$ $\mathcal{E}_{\tau}^{\mathcal{R}, \Lambda}(\cdot) / \mathcal{N}_{\tau}(\cdot)$ and $\left.\mathcal{G}_{\mathcal{S}}^{\mathcal{R}_{2}}(\cdot)=\mathcal{E}_{\mathcal{S}}^{\mathcal{R}_{2}}(\cdot) / \mathcal{N}_{\mathcal{S}}(\cdot)\right)$ is (resp. are) a sheaf (resp. presheaves) of differential algebras and a sheaf (resp. presheaves) of modules over the factor ring of generalized constants $\widetilde{\mathbb{C}}=\mathcal{E}_{M}(\mathbb{C}) / \mathcal{N}(\mathbb{C})$.

For any regular subspace $\mathcal{R}$ of $\mathbb{R}_{+}^{\mathbb{N}}$, the notion of support of a section $f \in \mathcal{G}^{\mathcal{R}}(\Omega)$ makes sense since $\mathcal{G}^{\mathcal{R}}(\cdot)$ is a sheaf. We denote by $\mathcal{G}_{C}^{\mathcal{R}}(\Omega)$ the subset of $\mathcal{G}^{\mathcal{R}}(\Omega)$ of elements with compact support. Note that every $f \in \mathcal{G}_{C}^{\mathcal{R}}$ has a representative $\left(f_{\varepsilon}\right)_{\varepsilon}$ such that each $f_{\varepsilon}$ has the same compact support.

EXAMPLE 2.3.

(i) Taking $\mathcal{R}=\mathbb{R}_{+}^{\mathbb{N}}$ (resp. $\mathcal{R}=\mathbb{R}_{+}^{\mathbb{N}}$ and $\Lambda=\mathbb{R}_{+}^{\mathbb{N}}$ ), we recover the sheaf $\mathcal{G}(\cdot)=\mathcal{G}^{\mathbb{R}_{+}^{\mathbb{N}}(\cdot)}$

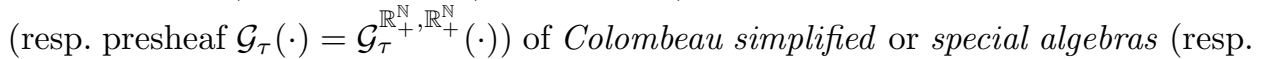
of tempered generalized functions) [3, 4, 14, 16]. Taking $\mathcal{R}_{2}=\mathbb{R}_{+}^{\mathbb{N}^{2}}$, we obtain the presheaf $\mathcal{G}_{\mathcal{S}}(\cdot)=\mathcal{G}_{\mathcal{S}}^{\mathbb{R}_{+}^{\mathbb{N}^{2}}}(\cdot)$ of algebras of rapidly decreasing generalized functions 8, 15, 16.

(ii) Taking $\mathcal{R}=\mathcal{B}$, introduced in Example 2.2, we obtain the sheaf of $\mathcal{G}^{\infty}$-generalized functions 14 .

Notation 2.4. We set $\mathcal{E}_{M}(\cdot)=\mathcal{E}_{M}^{\mathbb{R}_{+}^{\mathbb{N}}}(\cdot), \mathcal{E}_{\tau}(\cdot)=\mathcal{E}_{\tau}^{\mathbb{R}_{+}^{\mathbb{N}}, \mathbb{R}_{+}^{\mathbb{N}}}(\cdot)$ and $\mathcal{E}_{\mathcal{S}}(\cdot)=\mathcal{E}_{\mathcal{S}}^{\mathbb{R}_{+}^{\mathbb{N}^{2}}}(\cdot) ;\left[\left(f_{\varepsilon}\right)_{\varepsilon}\right]$ (resp. $\left.\left[\left(f_{\varepsilon}\right)_{\varepsilon}\right]_{\tau},\left[\left(f_{\varepsilon}\right)_{\varepsilon}\right]_{\mathcal{S}}\right)$ denotes the class of $\left(f_{\varepsilon}\right)_{\varepsilon}$ in $\mathcal{G}(\cdot)\left(\operatorname{resp} . \mathcal{G}_{\tau}(\cdot), \mathcal{G}_{\mathcal{S}}(\cdot)\right)$.

In this paper, we consider mainly the following two examples of regular spaces. 
EXAMPLE 2.5 (generalized functions with sublinear asymptotic growth, 5, 7]). Set

$$
\begin{aligned}
& \mathcal{L}_{0}=\left\{N \in \mathbb{R}_{+}^{\mathbb{N}} \mid \lim _{l \rightarrow+\infty}(q(l) / l)=0\right\}, \\
& \mathcal{L}_{a}=\left\{N \in \mathbb{R}_{+}^{\mathbb{N}} \mid \limsup _{l \rightarrow+\infty}(N(l) / l)<a\right\}(a>0) .
\end{aligned}
$$

(i) The corresponding sheaves $\mathcal{G}^{\mathcal{L}_{a}}(\cdot)$ of algebras are called the sheaves (resp. presheaves of temperate) generalized functions with sublinear asymptotic growth.

(ii) Define analogously

$$
\begin{aligned}
& \mathcal{E}_{\tau}^{\mathcal{L}_{a}}(\Omega)=\left\{\left(f_{\varepsilon}\right) \in \mathcal{O}_{M}(\Omega)^{(0,1]} \mid \exists N \in \mathcal{L}_{a}, \exists q \in \mathbb{N}, \quad \forall l \in \mathbb{N}, \quad \mu_{-q, l}\left(f_{\varepsilon}\right)=\mathrm{O}\left(\varepsilon^{-N(l)}\right)\right\}, \\
& \mathcal{E}_{\mathcal{S}}^{\mathcal{L}_{a}}(\Omega)=\left\{\left(f_{\varepsilon}\right)_{\varepsilon} \in \mathcal{S}(\Omega)^{(0,1]} \mid \exists N \in \mathcal{L}_{a}, \forall q \in \mathbb{N}, \forall l \in \mathbb{N}, \quad \mu_{q, l}\left(f_{\varepsilon}\right)=\mathrm{O}\left(\varepsilon^{-N(l)}\right)\right\} .
\end{aligned}
$$

The presheaves $\mathcal{G}_{\tau}^{\mathcal{L}_{a}}(\cdot)=\mathcal{E}_{\tau}^{\mathcal{L}_{a}}(\cdot) / \mathcal{N}_{\mathcal{S}}(\cdot)\left(\operatorname{resp} . \mathcal{G}_{\mathcal{S}}^{\mathcal{L}_{a}}(\cdot)=\mathcal{E}_{\mathcal{S}}^{\mathcal{L}_{a}}(\cdot) / \mathcal{N}_{\mathcal{S}}(\cdot)\right)$ are called the presheaves of temperate (resp. rapidly decreasing) generalized functions with sublinear asymptotic growth.

In other words, for $\mathcal{E}_{\tau}^{\mathcal{L}_{a}}(\cdot)$, we take $\mathcal{R}=\mathcal{L}_{a}$ and $\Lambda=\mathcal{B}$ (the set of bounded sequences). For $\mathcal{E}_{\mathcal{S}}^{\mathcal{L}_{a}}(\cdot)$, we take

$$
\mathcal{R}_{2}=\left\{N_{2} \in \mathbb{R}_{+}^{\mathbb{N}^{2}} \mid \exists N \in \mathcal{L}_{a}, \forall(q, l) \in \mathbb{N}^{2}, \quad N_{2}(q, l)=N(l)\right\} .
$$

We refer the reader to [6] for a thorough study of regular subspaces of $\mathcal{G}_{\mathcal{S}}(\cdot)$.

2.2. Some embeddings [6, 13]. As we need in the sequel some results related to the embeddings of classical spaces into spaces of nonlinear generalized functions, we collect them here without proof.

(i) The canonical map $\sigma: \mathrm{C}^{\infty}(\Omega) \rightarrow \mathcal{G}^{\mathcal{R}}(\Omega), f \rightarrow\left[\left(f_{\varepsilon}\right)_{\varepsilon}\right]$, with $f_{\varepsilon}=f$ for all $\varepsilon \in(0,1]$, is an embedding of differential algebras. For the embedding of $\mathcal{D}^{\prime}(\Omega)$ into $\mathcal{G}(\Omega)$, consider $\rho \in \mathcal{S}\left(\mathbb{R}^{d}\right)$ such that

$$
\int \rho(x) \mathrm{d} x=1, \quad \int x^{m} \rho(x) \mathrm{d} x=0 \text { for all } m \in \mathbb{N}^{d} \backslash\{0\} .
$$

Choose $\chi \in \mathcal{D}\left(\mathbb{R}^{d}\right)$ such that $0 \leq \chi \leq 1, \chi \equiv 1$ on a neighborhood of 0 . Define

$$
\forall \varepsilon \in(0,1], \forall x \in \mathbb{R}^{d}, \quad \theta_{\varepsilon}(x)=\varepsilon^{-d} \rho(x / \varepsilon) \chi(|\ln \varepsilon| x) .
$$

It is easily seen that $\left(\theta_{\varepsilon}\right)_{\varepsilon}$ satisfy, for all $k \in \mathbb{N}$,

$$
\int \theta_{\varepsilon}(x) \mathrm{d} x=1+\mathrm{O}\left(\varepsilon^{k}\right), \quad \forall m \in \mathbb{N}^{d} \backslash\{0\}, \int x^{m} \theta_{\varepsilon}(x) \mathrm{d} x=\mathrm{O}\left(\varepsilon^{k}\right) .
$$

Finally, consider $\left(\kappa_{\varepsilon}\right)_{\varepsilon} \in\left(\mathcal{D}\left(\mathbb{R}^{d}\right)\right)^{(0,1]}$ such that

$$
\forall \varepsilon \in(0,1], 0 \leq \kappa_{\varepsilon} \leq 1, \kappa_{\varepsilon} \equiv 1 \text { on }\left\{x \in \Omega \mid d\left(x, \mathbb{R}^{d} \backslash \Omega\right) \geq \varepsilon \text { and } d(x, 0) \leq 1 / \varepsilon\right\} .
$$

With these ingredients, the map

$$
\iota: \mathcal{D}^{\prime}(\Omega) \rightarrow \mathcal{G}(\Omega), \quad T \mapsto\left(\kappa_{\varepsilon} T * \theta_{\varepsilon}\right)_{\varepsilon}+\mathcal{N}(\Omega)
$$

is an embedding of $\mathcal{D}^{\prime}(\Omega)$ into $\mathcal{G}(\Omega)$ such that $\iota_{\mid \mathrm{C}^{\infty}(\Omega)}=\sigma$. Note that the additional cutoff $\left(\kappa_{\varepsilon}\right)_{\varepsilon}$ is not needed if $\Omega=\mathbb{R}^{d}$.

(ii) The canonical map $\sigma_{\mathcal{S}}: \mathcal{S}\left(\mathbb{R}^{d}\right) \rightarrow \mathcal{G}_{\mathcal{S}}\left(\mathbb{R}^{d}\right), f \mapsto\left[\left(f_{\varepsilon}\right)_{\varepsilon}\right]_{\mathcal{S}}$, with $f_{\varepsilon}=f$ for all $\varepsilon \in(0,1]$, is an embedding of differential algebras. Moreover, the image of $\sigma_{\mathcal{S}}$ is included in $\mathcal{G}_{\mathcal{S}}^{\mathcal{R}_{2}}\left(\mathbb{R}^{d}\right)$ 
for any regular subset of $\mathcal{R}_{2} \subset \mathbb{R}_{+}^{\mathbb{N}^{2}}$. The space of rapidly decreasing distributions $\mathcal{O}_{C}^{\prime}\left(\mathbb{R}^{d}\right)$ is embedded into $\mathcal{G}_{\mathcal{S}}\left(\mathbb{R}^{d}\right)$, via the map

$$
\iota_{\mathcal{S}}: \mathcal{O}_{C}^{\prime}\left(\mathbb{R}^{d}\right) \rightarrow \mathcal{G}_{\mathcal{S}}\left(\mathbb{R}^{d}\right), u \mapsto\left[\left(u * \rho_{\varepsilon}\right)_{\varepsilon}\right]_{\mathcal{S}}
$$

(with $\rho_{\varepsilon}(\cdot)=\varepsilon^{-d} \rho(\cdot / \varepsilon)$, where $\rho$ satisfies 2 ). This map is a linear embedding which commutes with partial derivatives and satisfies $\iota_{\mathcal{S} \mid \mathcal{S}\left(\mathbb{R}^{d}\right)}=\sigma_{\mathcal{S}}$.

(iii) The canonical map $\sigma_{\tau}: \mathcal{O}_{M}\left(\mathbb{R}^{d}\right) \rightarrow \mathcal{G}_{\tau}^{R}\left(\mathbb{R}^{d}\right), f \mapsto\left[(f)_{\varepsilon}\right]_{\tau}$, with $f_{\varepsilon}=f$ for all $\varepsilon \in(0,1]$, is an embedding of differential algebras. The map

$$
\iota_{\tau}: \mathcal{S}^{\prime}\left(\mathbb{R}^{d}\right) \rightarrow \mathcal{G}_{\tau}\left(\mathbb{R}^{d}\right), \quad u \mapsto\left(u * \rho_{\varepsilon}\right)_{\varepsilon}+\mathcal{N}_{\tau}\left(\mathbb{R}^{d}\right)
$$

with $\rho_{\varepsilon}$ defined as above is an embedding of differential vector spaces which commutes with partial derivatives. Moreover, $\iota_{\tau \mid \mathcal{O}_{C}\left(\mathbb{R}^{d}\right)}=\sigma_{\tau}$, where

$$
\mathcal{O}_{C}(\Omega)=\left\{f \in \mathrm{C}^{\infty}(\Omega) \mid \exists q \in \mathbb{N}, \forall l \in \mathbb{N}, \quad \mu_{-q, l}(f)<+\infty\right\} .
$$

\subsection{Units for convolution in spaces of generalized functions with sublin-} ear asymptotic growth. We recall here that convolution has no unit in $\mathcal{G}\left(\mathbb{R}^{d}\right.$ ) (resp. $\left.\mathcal{G}_{\mathcal{S}}\left(\mathbb{R}^{d}\right), \mathcal{G}_{\tau}\left(\mathbb{R}^{d}\right)\right)$. We are going to show that such units exist in some regular subspaces.

Definition 2.6. Consider a real number a greater than 1 and $\rho \in \mathcal{S}\left(\mathbb{R}^{d}\right)$ which satisfies (2). The net

$$
\left(\rho_{\varepsilon^{a}}(\cdot)\right)_{\varepsilon}=\left(\varepsilon^{-a d} \rho\left(\cdot / \varepsilon^{a}\right)\right)_{\varepsilon}
$$

is called an accelerated $\delta$-net.

This definition is justified by the following:

TheOREM 2.7. Consider $a \in \mathbb{R}_{+} \backslash\{0\}$ and $\rho \in \mathcal{S}\left(\mathbb{R}^{d}\right)$ which satisfies 2).

(i) Choose $\chi \in \mathcal{D}\left(\mathbb{R}^{d}\right)$ such that $0 \leq \chi \leq 1, \chi \equiv 1$ on a neighborhood of 0 and define $\left(\theta_{\varepsilon^{a}}\right)_{\varepsilon}$ by $\theta_{\varepsilon^{a}}(\cdot)=\varepsilon^{-a d} \rho\left(\cdot / \varepsilon^{a}\right) \chi(|\ln \varepsilon| \cdot)$ for $\varepsilon \in(0,1]$. For any $\left(g_{\varepsilon}\right)_{\varepsilon} \in \mathcal{E}_{M}^{\mathcal{L}_{a}}\left(\mathbb{R}^{d}\right)$, we have

$$
\left(g_{\varepsilon} * \theta_{\varepsilon^{a}}-g_{\varepsilon}\right)_{\varepsilon} \in \mathcal{N}\left(\mathbb{R}^{d}\right) .
$$

(ii) For any $\left(g_{\varepsilon}\right)_{\varepsilon} \in \mathcal{E}_{\mathcal{S}}^{\mathcal{L}_{a}}\left(\mathbb{R}^{d}\right)\left(\right.$ resp. $\left.\mathcal{E}_{\tau}^{\mathcal{L}_{a}}\left(\mathbb{R}^{d}\right)\right)$, we have

$$
\left(g_{\varepsilon} * \rho_{\varepsilon^{a}}-g_{\varepsilon}\right)_{\varepsilon} \in \mathcal{N}_{\mathcal{S}}\left(\mathbb{R}^{d}\right) \quad\left(\operatorname{resp} . \mathcal{N}_{\tau}\left(\mathbb{R}^{d}\right)\right) .
$$

Relation (5) (resp. (6) ) shows that $\left[\left(\theta_{\varepsilon^{a}}\right)_{\varepsilon}\right]$ (resp. $\left.\left[\left(\rho_{\varepsilon^{a}}\right)_{\varepsilon}\right]_{\mathcal{S}},\left[\left(\rho_{\varepsilon^{a}}\right)_{\varepsilon}\right]_{\tau}\right)$ plays the role of unit for convolution in $\mathcal{G}^{\mathcal{L}_{a}}\left(\mathbb{R}^{d}\right)\left(\operatorname{resp} . \mathcal{G}_{\mathcal{S}}^{\mathcal{L}_{a}}\left(\mathbb{R}^{d}\right), \mathcal{G}_{\tau}^{\mathcal{L}_{a}}\left(\mathbb{R}^{d}\right)\right)$.

Proof. We shall detail the proof of (i) and comment on the differences in the proof of (ii). (i) It is easily seen that $\left(\theta_{\varepsilon^{a}}\right)_{\varepsilon}$ satisfy, analogously to (4),

$$
\left(\int \theta_{\varepsilon^{a}}(x) \mathrm{d} x-1\right)_{\varepsilon} \in \mathcal{N}(\mathbb{R}) ; \quad \forall m \in \mathbb{N}^{d} \backslash\{0\}, \quad\left(\int x^{m} \theta_{\varepsilon}(x) \mathrm{d} x\right)_{\varepsilon} \in \mathcal{N}(\mathbb{R}) .
$$

Consider $r>0$ such that $\operatorname{supp} \theta \subset B(0, r)$. Fix $\left(g_{\varepsilon}\right)_{\varepsilon} \in \mathcal{E}_{M}^{\mathcal{L}_{a}}\left(\mathrm{C}^{\infty}\left(\mathbb{R}^{d}\right)\right), K$ a compact set of $\mathbb{R}^{d}$ and set $\Delta_{\varepsilon}=g_{\varepsilon} * \theta_{\varepsilon^{a}}-g_{\varepsilon}$ for $\varepsilon \in(0,1)$. Writing $\int \theta_{\varepsilon^{a}}(x) \mathrm{d} x=1+\mathcal{N}_{\varepsilon}$ with $\left(\mathcal{N}_{\varepsilon}\right)_{\varepsilon} \in \mathcal{N}(\mathbb{R})$, we get 


$$
\Delta_{\varepsilon}(y)=\int g_{\varepsilon}(y-x) \theta_{\varepsilon^{a}}(x) \mathrm{d} x-g_{\varepsilon}(y)=\int\left(g_{\varepsilon}(y-x)-g_{\varepsilon}(y)\right) \theta_{\varepsilon^{a}}(x) \mathrm{d} x+\mathcal{N}_{\varepsilon} g_{\varepsilon}(y) .
$$

The integration is performed on the compact set $B(0, r /|\ln \varepsilon|)$ which contains $\operatorname{supp} \theta_{\varepsilon}$.

There exists a compact set $K^{\prime}$ such that $y+B(0,1) \subset K^{\prime}$ for all $y \in K$, and a sequence $N \in \mathcal{L}_{a}$ such that

$$
\forall i \in \mathbb{N}, \quad p_{K^{\prime}, i}\left(g_{\varepsilon}\right)=\mathrm{O}\left(\varepsilon^{-N(i)}\right) .
$$

Note that $\left(\mathcal{N}_{\varepsilon} g_{\varepsilon}(\cdot)\right)_{\varepsilon}$ belongs clearly to $\mathcal{N}\left(\mathbb{R}^{d}\right)$. Thus we focus on estimating

$$
\Delta_{\varepsilon}^{1}(y)=\int\left(g_{\varepsilon}(y-x)-g_{\varepsilon}(y)\right) \theta_{\varepsilon}(x) \mathrm{d} x .
$$

Let $m$ be a positive integer. As $\limsup _{i \rightarrow+\infty}(N(i) / i)<a$, we get $\lim _{i \rightarrow+\infty}(a i-N(i))=$ $+\infty$ and the existence of an integer $k$ such that $k-N(k)>m$. Taylor's formula gives

$$
g_{\varepsilon}(y-x)-g_{\varepsilon}(y)=\sum_{i=1}^{k-1} \frac{1}{i !} D^{i} g_{\varepsilon}(y) \cdot(-x)^{i}+\int_{0}^{1} \frac{(1-t)^{k-1}}{(k-1) !} D^{k} g_{\varepsilon}^{(k)}(y-t x) \cdot(-x)^{k} \mathrm{~d} t .
$$

Thus

$$
\Delta_{\varepsilon}^{1}(y)=P_{\varepsilon}(k, y)+R_{\varepsilon}(k, y)
$$

where

$$
P_{\varepsilon}(k, y)=\int \sum_{i=1}^{k-1} \frac{1}{i !} D^{i} g_{\varepsilon}(y) \cdot(-x)^{i} \theta_{\varepsilon^{a}}(x) \mathrm{d} x
$$

and

$$
R_{\varepsilon}(k, y)=\iint_{0}^{1} \frac{(1-t)^{k-1}}{(k-1) !} D^{k} g_{\varepsilon}^{(k)}(y-t x) \cdot(-x)^{k} \mathrm{~d} t \theta_{\varepsilon^{a}}(x) \mathrm{d} x .
$$

According to 77 we have $\left(\int x^{j} \theta_{\varepsilon}(x) \mathrm{d} x\right)_{\varepsilon} \in \mathcal{N}(\mathbb{R})$ for all $j \in \mathbb{N}$. Consequently

$$
\sup _{y \in K}\left|P_{\varepsilon}(k, y)\right|=\mathrm{O}\left(\varepsilon^{m}\right) .
$$

Using the definition of $\theta_{\varepsilon^{a}}$, and setting $v=x / \varepsilon^{a}$, we get

$$
R_{\varepsilon}(k, y)=\varepsilon^{a k} \int_{B\left(0, \frac{r}{\varepsilon^{a} \ln \varepsilon}\right)} \int_{0}^{1} \frac{(1-t)^{k-1}}{(k-1) !} D^{k} g_{\varepsilon}^{(k)}\left(y-\varepsilon^{a} t v\right) \cdot(-v)^{k} \mathrm{~d} t \rho(v) \chi\left(\varepsilon^{a} \ln \varepsilon v\right) \mathrm{d} v .
$$

For $(t, v) \in[0,1] \times B\left(0, r /\left(\varepsilon^{a}|\ln \varepsilon|\right)\right)$, we have $y-\varepsilon^{a} t v \in y+B(0,1)$ for $\varepsilon$ small enough. Then, for $y \in K, y-\varepsilon^{a} t v$ lies in $K^{\prime}$ for $(t, v)$ in the domain of integration. It follows

$$
\begin{aligned}
\left|R_{\varepsilon}(k, y)\right| \leq \frac{\varepsilon^{a k}}{(k-1) !} p_{K^{\prime}, k}\left(g_{\varepsilon}\right) \int_{B\left(0, \frac{r}{\varepsilon^{a}|\ln \varepsilon|}\right)}|v|^{k}|\rho(v)| \mathrm{d} v & \leq C \varepsilon^{a k} p_{K^{\prime}, k}\left(g_{\varepsilon}\right) \\
& \leq C \varepsilon^{a k-N(k)}=\mathrm{O}\left(\varepsilon^{m}\right) .
\end{aligned}
$$

The constant $C>0$ depends only on the integer $k$ and on $\rho$. Summing up all results, we get $\sup _{y \in K}\left|\Delta_{\varepsilon}(y)\right|=\mathrm{O}\left(\varepsilon^{m}\right)$.

As $\left(\Delta_{\varepsilon}\right)_{\varepsilon} \in \mathcal{E}_{M}\left(\mathbb{R}^{d}\right)$ and $p_{K, 0}\left(\Delta_{\varepsilon}\right)=\mathrm{O}\left(\varepsilon^{m}\right)$ for all $m>0$ and $K \subset \subset \mathbb{R}$, we conclude that $\left(\Delta_{\varepsilon}\right)_{\varepsilon} \in \mathcal{N}\left(\mathbb{R}^{d}\right)$, without estimating the derivatives, by using theorem 1.2 .3 of [11].

(ii) The main tools for the proof of both cases are, as for $(i)$, the Taylor formula and the nature of the regular spaces $\mathcal{L}_{a}$. Nonetheless, let us quote some differences: On the one 
hand, both cases differ from $(i)$ by the global estimates they need; On the other hand, the construction of the accelerated $\delta$-net is simpler. For the case of $\mathcal{E}_{\mathcal{S}}^{\mathcal{L}_{a}}\left(\mathbb{R}^{d}\right)$, the detailed proof can be found in 7]. For the case of $\mathcal{E}_{\tau}^{\mathcal{L}_{a}}\left(\mathbb{R}^{d}\right)$, note that the independence of $q$ with respect to the derivative index $l$ in the definition of $\mathcal{E}_{\tau}^{\mathcal{L}_{a}}\left(\mathbb{R}^{d}\right)$ plays a big part in the result (see (1)).

REMARK 2.8. In addition, we have a quasi-uniqueness result in the following sense: For every accelerated $\delta$-net $\left(\rho_{\varepsilon^{a}}(\cdot)\right)_{\varepsilon}$, one has $\rho_{\varepsilon^{a}} \rightarrow \delta$ as $\varepsilon \rightarrow 0$. Thus, all the units defined as above are associated. This does not violate the usual uniqueness of units for internal laws, since $\left(\theta_{\varepsilon^{a}}\right)_{\varepsilon}$ does not belongs to $\mathcal{E}_{M}^{\mathcal{L}_{a}}\left(\mathbb{R}^{d}\right)$. The same holds for $\left(\rho_{\varepsilon^{a}}\right)_{\varepsilon}$ defined as in (ii).

\section{Kernel theorems}

3.1. Generalized integral operators. From now on $m$ and $n$ are two strictly positive integers.

Definition 3.1. Let $H$ be in $\mathcal{G}\left(\mathbb{R}^{m+n}\right)$ (resp. $\mathcal{G}_{\tau}\left(\mathbb{R}^{m+n}\right)$ ). The integral operator of kernel $H$ is the map $\widetilde{H}$ defined by

$$
\widetilde{H}: \mathcal{G}_{C}\left(\mathbb{R}^{n}\right) \rightarrow \mathcal{G}\left(\mathbb{R}^{m}\right)\left(\operatorname{resp} . \mathcal{G}_{\mathcal{S}}\left(\mathbb{R}^{n}\right) \rightarrow \mathcal{G}_{\tau}\left(\mathbb{R}^{m}\right)\right), \quad f \mapsto \widetilde{H}(f)=\int H(\cdot, y) f(y) \mathrm{d} y
$$

The integral, in the previous definition, is well defined by means of representatives $\left(H_{\varepsilon}\right)_{\varepsilon}$ and $\left(f_{\varepsilon}\right)_{\varepsilon}$ of $H$ and $f$ respectively [3]. Indeed, for the case of $H \in \mathcal{G}\left(\mathbb{R}^{m+n}\right)$ and $f \in \mathcal{G}_{C}\left(\mathbb{R}^{n}\right)$, we have

$$
\widetilde{H}(f)=\left[\left(x \mapsto \int_{W} H_{\varepsilon}(x, y) f_{\varepsilon}(y) \mathrm{d} y\right)_{\varepsilon}\right]
$$

where $W$ is an open neighborhood of $\operatorname{supp} f$. Note that, in [2] and [8], the generalized function $H$ has to be properly supported since $\widetilde{H}$ acts on $\mathcal{G}\left(\mathbb{R}^{n}\right)$. For the case of $H$ in $\mathcal{G}_{\tau}\left(\mathbb{R}^{m+n}\right)$, the net $\left(y \mapsto H_{\varepsilon}(x, y) f_{\varepsilon}(y)\right)_{\varepsilon}$ belongs to $\mathcal{E}_{\mathcal{S}}\left(\mathbb{R}^{n}\right)$ for all $x$ in $\mathbb{R}^{m}$. Thus, the integral $\int H_{\varepsilon}(x, y) f_{\varepsilon}(y) \mathrm{d} y$ exists for all $\varepsilon \in(0,1]$. Moreover, the net $\left(x \mapsto \int H_{\varepsilon}(x, y) f_{\varepsilon}(y) \mathrm{d} y\right)_{\varepsilon}$ belongs to $\mathcal{E}_{\tau}\left(\mathbb{R}^{n}\right)$ and its class defines the element $\widetilde{H}(f)$ of $\mathcal{G}_{\tau}\left(\mathbb{R}^{m}\right)$.

In both cases, it can be easily seen that the map $\widetilde{H}$ is a linear map of $\widetilde{\mathbb{C}}$-modules, continuous for the respective sharp topologies of the spaces under consideration. We refer the reader to [17, 18, for the sharp topology and to [2] for additional comments on continuity for integral operators acting on the usual Colombeau algebras. The map which associates $\widetilde{H}$ to $H$ is injective. Indeed, for the case of $H \in \mathcal{G}\left(\mathbb{R}^{m+n}\right)$ acting on $\mathcal{G}_{C}\left(\mathbb{R}^{n}\right)$, one adapts the proofs given in [2] or [8] for the case of $H \in \mathcal{G}\left(\mathbb{R}^{m+n}\right)$ properly supported acting on $\mathcal{G}\left(\mathbb{R}^{n}\right)$. The case of $H \in \mathcal{G}_{\tau}\left(\mathbb{R}^{m+n}\right)$ is treated in [7].

3.2. Extension of linear maps. We turn to the definition and the properties of the nets of linear maps used in the main theorems. We have to distinguish the two cases under consideration. This is due, in particular, to the topological nature of $\mathcal{D}\left(\mathbb{R}^{n}\right)$. We recall that $\mathcal{D}(\Omega)$ can be considered as the inductive limit of

$$
\mathcal{D}_{j}(\Omega)=\mathcal{D}_{K_{j}}(\Omega)=\left\{f \in \mathcal{D}(\Omega) \mid \operatorname{supp} f \subset K_{j}\right\},
$$


where $\left(K_{j}\right)_{j \in \mathbb{N}}$ is an increasing sequence of compact sets exhausting $\Omega$, with $K_{j} \subset \stackrel{\circ}{K_{j+1}}$ and $\mathcal{D}_{j}(\Omega)$ is endowed with the topology given by the family of seminorms $\left(p_{j, l}\right)_{l \in \mathbb{N}}$ defined by $p_{j, l}(f)=\sup _{x \in K_{j},|\alpha| \leq l}\left|\partial^{\alpha} f(x)\right|$. The topology on $\mathcal{D}(\Omega)$ does not depend on the particular choice of the sequence $\left(K_{j}\right)_{j \in \mathbb{N}}$.

Definition $3.2([\underline{5}])$. Let $\left(L_{\varepsilon}\right)_{\varepsilon} \in \mathcal{L}\left(\mathcal{D}\left(\mathbb{R}^{n}\right), \mathrm{C}^{\infty}\left(\mathbb{R}^{m}\right)\right)^{(0,1]}$ be a net of linear maps.

(i) We say that $\left(L_{\varepsilon}\right)_{\varepsilon}$ is continuously moderate (resp. negligible) if

$\forall j \in \mathbb{N}, \quad \forall K \subset \subset \mathbb{R}^{m}, \forall l \in \mathbb{N}, \exists\left(C_{\varepsilon}\right)_{\varepsilon} \in \mathcal{X}_{M}\left(\mathbb{R}_{+}\right)\left(\operatorname{resp} . \mathcal{N}\left(\mathbb{R}_{+}\right)\right)$,

$\exists l^{\prime} \in \mathbb{N}, \forall f \in \mathcal{D}_{j}\left(\mathbb{R}^{n}\right), p_{K, l}\left(L_{\varepsilon}(f)\right) \leq C_{\varepsilon} p_{j, l^{\prime}}(f)$, for $\varepsilon$ small enough.

(ii) Let $(b, c)$ be in $[0,+\infty] \times \mathbb{R}^{+}$. We say that $\left(L_{\varepsilon}\right)_{\varepsilon}$ is $\mathcal{L}_{b, c^{-}}$strongly continuously moderate if: $\forall j \in \mathbb{N}, \forall K \subset \subset \mathbb{R}^{m}$,

$\exists \lambda \in \mathbb{N}^{\mathbb{N}}$ with $\limsup _{l \rightarrow+\infty}(\lambda(l) / l) \leq b, \exists r \in \mathbb{R}_{+}^{\mathbb{N}}$ with $\limsup _{l \rightarrow+\infty}(r(l) / l) \leq c$,

$\forall l \in \mathbb{N}, \exists C \in \mathbb{R}_{+}, \forall f \in \mathcal{D}_{j}\left(\mathbb{R}^{n}\right), p_{K, l}\left(L_{\varepsilon}(f)\right) \leq C \varepsilon^{-r(l)} p_{j, \lambda(l)}(f)$, for $\varepsilon$ small enough.

Definition $3.3([7])$. Let $\left(L_{\varepsilon}\right)_{\varepsilon} \in \mathcal{L}\left(\mathcal{S}\left(\mathbb{R}^{n}\right), \mathcal{O}_{M}\left(\mathbb{R}^{m}\right)\right)^{(0,1]}$ be a net of linear maps.

(i) We say that $\left(L_{\varepsilon}\right)_{\varepsilon}$ is moderate (resp. negligible) if

$$
\begin{gathered}
\forall l \in \mathbb{N}, \exists\left(C_{\varepsilon}\right)_{\varepsilon} \in \mathcal{E}_{M}\left(\mathbb{R}_{+}\right) \quad\left(\operatorname{resp} . \mathcal{N}\left(\mathbb{R}_{+}\right)\right), \exists\left(p, q, l^{\prime}\right) \in \mathbb{N}^{3}, \\
\forall f \in \mathcal{S}\left(\mathbb{R}^{n}\right), \mu_{-p, l}\left(L_{\varepsilon}(f)\right) \leq C_{\varepsilon} \mu_{q, l^{\prime}}(f), \text { for } \varepsilon \text { small enough. }
\end{gathered}
$$

(ii) Let $(b, c)$ be in $[0,+\infty] \times \mathbb{R}_{+}$. We say that $\left(L_{\varepsilon}\right)_{\varepsilon}$ is $\mathcal{L}_{b, c^{-}}$strongly moderate if

$\exists \lambda \in \mathbb{N}^{\mathbb{N}}$ with $\lim \sup (\lambda(l) / l) \leq b, \exists r \in \mathbb{R}_{+}^{\mathbb{N}}$ with $\limsup (r(l) / l) \leq c$,

$$
\forall l \in \mathbb{N}, \exists C \in \mathbb{R}_{+}, \exists(p, q) \in \mathbb{N}^{2}, \forall f \in \mathcal{S}\left(\mathbb{R}^{n}\right), \mu_{-p, l}\left(L_{\varepsilon}(f)\right) \leq C \varepsilon^{-r(l)} \mu_{q, \lambda(l)}(f),
$$

for $\varepsilon$ small enough.

These nets give rise to linear maps acting between the respective generalized spaces. More precisely, we get the:

\section{Proposition $3.4([5,7])$.}

(i) Any continuously moderate net

$$
\left(L_{\varepsilon}\right)_{\varepsilon} \in \mathcal{L}\left(\mathcal{D}\left(\mathbb{R}^{n}\right), \mathrm{C}^{\infty}\left(\mathbb{R}^{m}\right)\right)^{(0,1]}\left(\operatorname{resp} . \mathcal{L}\left(\mathcal{S}\left(\mathbb{R}^{n}\right), \mathcal{O}_{M}\left(\mathbb{R}^{m}\right)\right)^{(0,1]}\right)
$$

can be extended in a map $L \in \mathcal{L}\left(\mathcal{G}_{C}\left(\mathbb{R}^{n}\right), \mathcal{G}\left(\mathbb{R}^{m}\right)\right)$ (resp. $\mathcal{L}\left(\mathcal{G}_{\mathcal{S}}\left(\mathbb{R}^{n}\right), \mathcal{G}_{\tau}\left(\mathbb{R}^{m}\right)\right)$ ) defined by $L(f)=\left(L_{\varepsilon}\left(f_{\varepsilon}\right)\right)_{\varepsilon}+\mathcal{N}\left(\mathbb{R}^{m}\right)$ (resp. $\mathcal{N}_{\tau}\left(\mathbb{R}^{m}\right)$ ) where $\left(f_{\varepsilon}\right)$ is any representative of $f$.

(ii) The extension $L$ depends only on the family $\left(L_{\varepsilon}\right)$ in the following sense: If $\left(N_{\varepsilon}\right)_{\varepsilon}$ is a net of negligible maps, then the extensions of $\left(L_{\varepsilon}\right)_{\varepsilon}$ and $\left(L_{\varepsilon}+N_{\varepsilon}\right)_{\varepsilon}$ are equal. The extension $L$ depends only on the family $\left(L_{\varepsilon}\right)$ in the following sense: If $\left(N_{\varepsilon}\right)_{\varepsilon}$ is a net of negligible maps, then the extensions of $\left(L_{\varepsilon}\right)_{\varepsilon}$ and $\left(L_{\varepsilon}+N_{\varepsilon}\right)_{\varepsilon}$ are equal.

(iii) If the family $\left(L_{\varepsilon}\right)_{\varepsilon}$ is moderate, with the assumption that the net of constants $\left(C_{\varepsilon}\right)_{\varepsilon}$ in (8) (resp. (9)) satisfies $C_{\varepsilon}=\mathrm{O}\left(\varepsilon^{-r(l)}\right)$ with $\limsup _{l \rightarrow+\infty}(r(l) / l)<c$, then $L\left(\mathcal{G}_{C}^{\infty}\left(\mathbb{R}^{n}\right)\right)$ (resp. $\left.L\left(\mathcal{G}_{\mathcal{S}}^{\infty}\left(\mathbb{R}^{n}\right)\right)\right)$ is included in $\mathcal{G}^{\mathcal{L}_{c}}\left(\mathbb{R}^{m}\right)$ (resp. $\left.\mathcal{G}_{\tau}^{\mathcal{L}_{c}}\left(\mathbb{R}^{m}\right)\right)$.

(iv) Let $(b, c)$ be in $\left(\mathbb{R}^{+}\right)^{3}$ and suppose that the net $\left(L_{\varepsilon}\right)_{\varepsilon}$ is $\mathcal{L}_{b, c}$-strongly continuously moderate. Then, for all $a>0, L\left(\mathcal{G}_{C}^{\mathcal{L}_{a}}\left(\mathbb{R}^{n}\right)\right)\left(\right.$ resp. $\left.\mathcal{G}_{\mathcal{S}}^{\mathcal{L}_{a}}\left(\mathbb{R}^{n}\right)\right)$ is included in $\mathcal{G}^{\mathcal{L}_{a b+c}}\left(\mathbb{R}^{m}\right)$ (resp. $\left.\mathcal{G}_{\tau}^{\mathcal{L}_{a b+c}}\left(\mathbb{R}^{n}\right)\right)$. 
Moreover, $L\left(\mathcal{G}_{C}^{\mathcal{L}_{0}}\left(\mathbb{R}^{n}\right)\right)$ (resp. $L\left(\mathcal{G}_{\mathcal{S}}^{\mathcal{L}_{0}}\left(\mathbb{R}^{n}\right)\right)$ ) is included in $\mathcal{G}^{\mathcal{L}_{c^{\prime}}}\left(\mathbb{R}^{m}\right)$ (resp. $\mathcal{G}_{\tau}^{\mathcal{L}_{c^{\prime}}}\left(\mathbb{R}^{m}\right)$ ) for any $c^{\prime}>c$, even if $b=+\infty$.

REMARK 3.5. Following [21, a linear map $L: \mathcal{D}\left(\mathbb{R}^{n}\right) \rightarrow \mathcal{G}\left(\mathbb{R}^{m}\right)$ (which is a similar situation to ours) is continuously moderate if, and only if, it is $r$-continuous and moderate in the following sense: $L$ admits a representative $\left(L_{\varepsilon}: \mathcal{D}\left(\mathbb{R}^{n}\right) \rightarrow \mathrm{C}^{\infty}\left(\mathbb{R}^{m}\right)\right)_{\varepsilon}$ formed by continuous linear maps, which are moderate (that is: $\left(L_{\varepsilon}(f)\right)_{\varepsilon} \in \mathcal{E}_{M}\left(\mathbb{R}^{m}\right)$, for all $f \in$ $\left.\mathcal{D}\left(\mathbb{R}^{n}\right)\right)$. This, in some sense, justifies the definition given here of continuously moderate nets.

\subsection{Kernel theorems}

TheOREm 3.6 (Schwartz kernel type theorem). Consider $(b, c) \in \mathbb{R}_{+}^{2}$. Let $\left(L_{\varepsilon}\right) \in$ $\mathcal{L}\left(\mathcal{D}\left(\mathbb{R}^{n}\right), \mathrm{C}^{\infty}\left(\mathbb{R}^{m}\right)\right)^{(0,1]}$ be a net of $\mathcal{L}_{b, c}$-strongly continuously moderate linear maps and $L \in \mathcal{L}\left(\mathcal{G}_{C}\left(\mathbb{R}^{n}\right), \mathcal{G}\left(\mathbb{R}^{m}\right)\right)$ its canonical extension. For any a $>0$, there exists $H_{L}$ in $\mathcal{G}^{\mathcal{L}_{2(a b+c)}\left(\mathbb{R}^{m+n}\right) \text { such that }}$

$$
\forall f \in \mathcal{G}_{C}^{\mathcal{L}_{a}}\left(\mathbb{R}^{n}\right), \quad L(f)=\int H_{L}(\cdot, y) f(y) \mathrm{d} y .
$$

TheOREM 3.7 (nuclear kernel type theorem). Consider $(b, c) \in \mathbb{R}_{+}^{2}$ and $\left(L_{\varepsilon}\right)_{\varepsilon} \in$ $\mathcal{L}\left(\mathcal{S}\left(\mathbb{R}^{n}\right), \mathcal{O}_{M}\left(\mathbb{R}^{m}\right)\right)^{(0,1]}$ a net of $\mathcal{L}_{b, c}$-strongly moderate linear maps. Denote by $L \in$ $\mathcal{L}\left(\mathcal{G}_{\mathcal{S}}\left(\mathbb{R}^{n}\right), \mathcal{G}_{\tau}\left(\mathbb{R}^{m}\right)\right)$ its canonical extension. For any a $>0$, there exists $H_{L}$ in $\mathcal{G}_{\tau}^{\mathcal{L}_{2(a b+c)}}\left(\mathbb{R}^{m+n}\right)$ such that

$$
\forall f \in \mathcal{G}_{\mathcal{S}}^{\mathcal{L}_{a}}\left(\mathbb{R}^{n}\right), \quad L(f)=\int H_{L}(\cdot, y) f(y) \mathrm{d} y .
$$

These results have exactly the same structure. In fact, the difference between them is somehow hidden but can be seen in the definition of moderate maps which takes into account the specific topological structure of the starting spaces (see Definitions 3.2 and 3.3. In both results, the parameter $a$ gives the size of the space on which the result holds, the parameter $b$ (resp. $c$ ) is related to the "regularity" of the net $\left(L_{\varepsilon}\right)_{\varepsilon}$, with respect to the derivative index $l$ in the family of semi-norms defining the topology (resp. to the parameter $\varepsilon$ ).

By using Proposition 3.4 (iii), we can obtain an analog of this result valid for more irregular nets of maps. We give it for the case of nuclear kernel type theorem.

TheOREM 3.8. Let $\left(L_{\varepsilon}\right)_{\varepsilon} \in \mathcal{L}\left(\mathcal{S}\left(\mathbb{R}^{n}\right), \mathcal{O}_{M}\left(\mathbb{R}^{m}\right)\right)^{(0,1]}$ be a net of moderate linear maps such that the net of constants $\left(C_{\varepsilon}\right)_{\varepsilon}$ in relation (9) satisfies $C_{\varepsilon}=\mathrm{O}\left(\varepsilon^{-r(l)}\right)$ with $r \in \mathcal{L}_{c}$ $(c>0)$. Then, the extension of $\left(L_{\varepsilon}\right)_{\varepsilon}$ admits an integral representation such that relation (10) holds for $f$ in $\mathcal{G}_{\mathcal{S}}^{\infty}\left(\mathbb{R}^{n}\right)$.

We turn now to the relationship with the classical isomorphism result. Consider

$$
\Lambda \in \mathcal{L}\left(\mathcal{D}\left(\mathbb{R}^{n}\right), \mathcal{D}^{\prime}\left(\mathbb{R}^{m}\right)\right)\left(\operatorname{resp} . \mathcal{L}\left(\mathcal{S}\left(\mathbb{R}^{n}\right), \mathcal{S}^{\prime}\left(\mathbb{R}^{m}\right)\right)\right)
$$

and define a net of linear mappings $\left(L_{\varepsilon}\right)_{\varepsilon}$ by

$$
L_{\varepsilon}: \mathcal{D}\left(\mathbb{R}^{n}\right)\left(\operatorname{resp} . \mathcal{S}\left(\mathbb{R}^{n}\right)\right) \rightarrow \mathrm{C}^{\infty}\left(\mathbb{R}^{m}\right), \quad f \mapsto \Lambda(f) * \varphi_{\varepsilon},
$$

where $\left(\varphi_{\varepsilon}\right)_{\varepsilon}$ is constructed as $\left(\theta_{\varepsilon}\right)_{\varepsilon}$ in $(3)$ (for the resp. case, $\varphi$ could be simply chosen in $\mathcal{D}\left(\mathbb{R}^{m}\right)$ satisfying (2p).

We have: 
Proposition 3.9.

(i) For all $\varepsilon \in(0,1], L_{\varepsilon}$ is continuous for the usual topologies of $\mathcal{D}\left(\mathbb{R}^{n}\right)$ (resp. $\mathcal{S}\left(\mathbb{R}^{n}\right)$ ) and $C^{\infty}\left(\mathbb{R}^{n}\right)$ (resp. $\mathcal{O}_{M}\left(\mathbb{R}^{m}\right)$ ) and the net $\left(L_{\varepsilon}\right)_{\varepsilon}$ is $(0, s)$-strongly moderate for any $s>1$.

(ii) From (i), the extension $L_{\Lambda}$ of the net $\left(L_{\varepsilon}\right)_{\varepsilon}$ admits a kernel $H_{L_{\Lambda}}$ of sublinear asymptotic growth. Furthermore, for all $f \in \mathcal{D}\left(\mathbb{R}^{n}\right)$ (resp. $\mathcal{S}\left(\mathbb{R}^{n}\right)$ ), $\iota(\Lambda(f)$ ) (resp. $\left.\iota_{\tau}(\Lambda(f))\right)$ is equal to $\widetilde{H}_{L}(f)$.

From Proposition 3.9, we immediately get that the following diagram is commutative

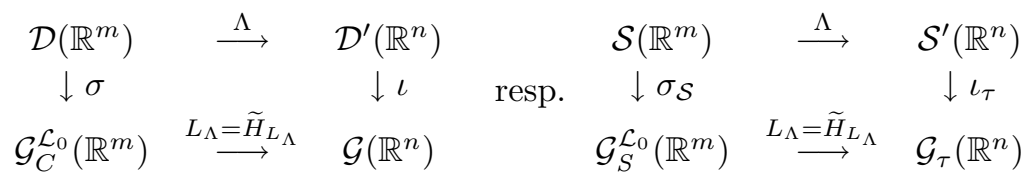

This shows that in this case, we transpose the classical result to the generalized framework (see [2] for further remarks on this subject). Note that Proposition 3.9 also implies that $\Lambda(f)$ and $\widetilde{H}_{L_{\Lambda}}(f)$ are equal in the generalized distribution sense 13 that is, for all $p \in \mathbb{N}$, and for the resp. case

$$
\forall g \in \mathcal{S}\left(\mathbb{R}^{m}\right), \quad\langle\Lambda(f), g\rangle-\int\left(\int H_{L_{\Lambda}, \varepsilon}(x, y) f(y) \mathrm{d} y\right) g(x) \mathrm{d} x=\mathrm{O}\left(\varepsilon^{p}\right),
$$

where $\left(H_{L_{\Lambda}, \varepsilon}\right)_{\varepsilon}$ is any representative of $H_{L_{\Lambda}}$. In particular, this result implies that $\Lambda(f)$ and $\widetilde{H}_{L_{\Lambda}}(f)$ are associated or weakly equal, i.e.

$$
\left(x \mapsto \int H_{L_{\Lambda}, \varepsilon}(x, y) f(y) \mathrm{d} y\right) \rightarrow \Lambda(f) \text { in } \mathcal{S}^{\prime}\left(\mathbb{R}^{m}\right) \text { as } \varepsilon \rightarrow 0 .
$$

Finally, let us mention that in [21], a kernel theorem is proved for $r$-continuous maps $L$ acting between $\mathcal{D}\left(\mathbb{R}^{n}\right.$ ) and $\mathcal{G}\left(\mathbb{R}^{m}\right)$ (see Remark 3.5). On the one hand, using the continuous and moderate representative of $L$, the result is more closely related to the classical one. For example, the proof can use the explicit form of the kernel of a continuous linear map from $\mathcal{D}\left(\mathbb{R}^{n}\right)$ to $\mathcal{D}\left(\mathbb{R}^{m}\right)$, as given by the classical Schwartz kernel theorem. We refer the reader to [21] for more details, and for additional properties of $\mathcal{G}^{\infty}$-type, specially concerning uniqueness, which are allowed by this framework. On the other hand, our results seem a little more general as they concern linear maps acting between spaces of generalized functions (even if these maps are particular ones in the sense they are given by representatives).

4. Proofs of the main results. We shall give the main ideas of the proof of Theorem 3.6. The proofs of Theorem 3.7 and 3.8 follow quite similar lines. In particular, Theorem 2.7 plays a major role, as seen at the end of the proof. To show different technics, we shall give the proof of Proposition 3.9 for the temperate case.

4.1. Proof of Theorem 3.6. Let us fix a net of mollifiers $\left(\varphi_{\varepsilon^{t}}\right)_{\varepsilon} \in\left(\mathcal{D}\left(\mathbb{R}^{m}\right)\right)^{(0,1]}$ (resp. $\left.\left(\psi_{\varepsilon^{a}}\right)_{\varepsilon} \in\left(\mathcal{D}\left(\mathbb{R}^{m}\right)\right)^{(0,1]}\right)$ satisfying conditions $[4]$, where $t \in \mathbb{R}_{+}$will be fixed latter. Set

$$
\psi_{\varepsilon^{a}, .}: \mathbb{R}^{n} \rightarrow \mathcal{D}\left(\mathbb{R}^{n}\right), \quad y \mapsto\left\{v \mapsto \psi_{\varepsilon^{a}}(y-v)\right\} .
$$


Consider

$$
H_{L, \varepsilon}: \mathbb{R}^{m+n} \rightarrow \mathbb{C}, \quad(x, y) \mapsto L_{\varepsilon}\left(\psi_{\varepsilon^{a}, y}\right) * \varphi_{\varepsilon^{t}}(x),
$$

which is well defined since $\varphi_{\varepsilon^{t}}$ is compactly supported. Classical regularity results imply that $H_{\varepsilon}$ is of class $\mathrm{C}^{\infty}$ for all $\varepsilon \in(0,1]$. A routine checking shows that $\left(H_{\varepsilon}\right)_{\varepsilon} \in \mathcal{E}_{M}\left(\mathbb{R}^{m+n}\right)$. A closer inspection (left to the reader) leads to

$$
\left(H_{\varepsilon}\right)_{\varepsilon} \in \mathcal{E}_{M}^{\mathcal{L}_{a b+c+t}}\left(\mathbb{R}^{m+n}\right) .
$$

We shall now prove that the operator $\widetilde{H}_{L}$ of kernel $\left[\left(H_{L, \varepsilon}\right)_{\varepsilon}\right]$ is the expected kernel operator. We fix $f \in \mathcal{G}_{C}\left(\mathbb{R}^{n}\right)$ and one of its representatives $\left(f_{\varepsilon}\right)_{\varepsilon}$ such that all $f_{\varepsilon}$ have the same compact support. We have to show that $L\left(\left[\left(f_{\varepsilon}\right)_{\varepsilon}\right]\right)=\left[\left(\widetilde{H}_{\varepsilon}\left(f_{\varepsilon}\right)\right)_{\varepsilon}\right]$.

Lemma 4.1. For all $\varepsilon \in(0,1]$, we have

$$
\widetilde{H}_{\varepsilon}\left(f_{\varepsilon}\right)(x)=\left(L_{\varepsilon}\left(\psi_{\varepsilon^{a}} * f_{\varepsilon}\right) * \varphi_{\varepsilon^{t}}\right)(x) .
$$

Proof. Fix $\varepsilon \in(0,1]$ and $x \in \mathbb{R}^{m}$. With the above notations, we have

$$
\begin{aligned}
\widetilde{H}_{\varepsilon}\left(f_{\varepsilon}\right)(x) & =\int_{\operatorname{supp} f}\left(\int_{\operatorname{supp} \varphi_{\varepsilon}} L_{\varepsilon}\left(\psi_{\varepsilon^{a}, y}\right)(x-\lambda) \varphi_{\varepsilon^{t}}(\lambda) \mathrm{d} \lambda\right) f_{\varepsilon}(y) \mathrm{d} y \\
& =\int_{\operatorname{supp} \varphi_{\varepsilon}} \int_{\operatorname{supp} f} L_{\varepsilon}\left(\psi_{\varepsilon^{a}, y}\right)(x-\lambda) \varphi_{\varepsilon^{t}}(\lambda) f_{\varepsilon}(y) \mathrm{d} \lambda \mathrm{d} y \\
& =\int\left(\int L_{\varepsilon}\left(\psi_{\varepsilon^{a}, y}\right)(x-\lambda) f_{\varepsilon}(y) \mathrm{d} y\right) \varphi_{\varepsilon^{t}}(\lambda) \mathrm{d} \lambda,
\end{aligned}
$$

the last two equalities being true by Fubini's theorem, each integral being calculated on a compact set. Then, it can be shown, using similar arguments as in [12] (Lemma 4.1.3) that

$$
\int L_{\varepsilon}\left(\psi_{\varepsilon^{a}, y}\right)(\xi) f_{\varepsilon}(y) \mathrm{d} y=L_{\varepsilon}\left(v \mapsto \int \psi_{\varepsilon^{a}}(y-v) f_{\varepsilon}(y) \mathrm{d} y\right)(\xi)=L_{\varepsilon}\left(\psi_{\varepsilon^{a}} * f_{\varepsilon}\right)(\xi) .
$$

Essentially, the integrals under consideration in the above equalities are integrals of continuous functions on compact sets and can be considered as limits of Riemann sums allowing the interchange of the integral and the linear map $L_{\varepsilon}$. Thus

$$
\widetilde{H}_{\varepsilon}\left(f_{\varepsilon}\right)(x)=\int L_{\varepsilon}\left(\psi_{\varepsilon^{a}} * f_{\varepsilon}\right)(x-\lambda) \varphi_{\varepsilon^{t}}(\lambda) \mathrm{d} \lambda=\left(L_{\varepsilon}\left(\psi_{\varepsilon^{a}} * f_{\varepsilon}\right) * \varphi_{\varepsilon^{t}}\right)(x)
$$

which completes the proof.

We suppose, in addition, that $\left(f_{\varepsilon}\right)_{\varepsilon}$ is in $\mathcal{E}^{\mathcal{L}_{a}}\left(\mathbb{R}^{n}\right)$. According to Lemma 4.1, we have for all $\varepsilon \in(0,1]$,

$$
\begin{aligned}
\widetilde{H}_{\varepsilon}\left(f_{\varepsilon}\right)-L_{\varepsilon}\left(f_{\varepsilon}\right) & =L_{\varepsilon}\left(\psi_{\varepsilon^{a}} * f_{\varepsilon}\right) * \varphi_{\varepsilon^{t}}-L_{\varepsilon}\left(f_{\varepsilon}\right) \\
& =L_{\varepsilon}\left(\psi_{\varepsilon^{a}} * f_{\varepsilon}\right) * \varphi_{\varepsilon^{t}}-L_{\varepsilon}\left(f_{\varepsilon}\right) * \varphi_{\varepsilon^{t}}+L_{\varepsilon}\left(f_{\varepsilon}\right) * \varphi_{\varepsilon^{t}}-L_{\varepsilon}\left(f_{\varepsilon}\right) \\
& =L_{\varepsilon}\left(\psi_{\varepsilon^{a}} * f_{\varepsilon}-f_{\varepsilon}\right) * \varphi_{\varepsilon^{t}}+L_{\varepsilon}\left(f_{\varepsilon}\right) * \varphi_{\varepsilon^{t}}-L_{\varepsilon}\left(f_{\varepsilon}\right) .
\end{aligned}
$$

As $\left(f_{\varepsilon}\right)_{\varepsilon} \in \mathcal{E}^{\mathcal{L}_{a}}\left(\mathbb{R}^{n}\right)$, we have $\left(L_{\varepsilon}\left(f_{\varepsilon}\right)\right)_{\varepsilon} \in \mathcal{E}^{\mathcal{L}_{a b+c}}\left(\mathbb{R}^{m}\right)$, because of the $\mathcal{L}_{b, c^{-}}$strongly continuously moderateness of $\left(L_{\varepsilon}\right)_{\varepsilon}$. We take $t=a b+c$ and obtain $\left(L_{\varepsilon}\left(f_{\varepsilon}\right) * \varphi_{\varepsilon^{t}}-L_{\varepsilon}\left(f_{\varepsilon}\right)\right)_{\varepsilon} \in$ $\mathcal{N}\left(\mathbb{R}^{m}\right)$. We also have $\left(\psi_{\varepsilon^{a}} * f_{\varepsilon}-f_{\varepsilon}\right)_{\varepsilon} \in \mathcal{N}\left(\mathbb{R}^{n}\right)$ by Theorem 2.7. Thus

$$
\left(L_{\varepsilon}\left(\psi_{\varepsilon^{a}} * f_{\varepsilon}-f_{\varepsilon}\right)\right)_{\varepsilon} \in \mathcal{N}\left(\mathbb{R}^{m}\right) .
$$


As $\left(\eta_{\varepsilon} * \varphi_{\varepsilon^{t}}\right)_{\varepsilon} \in \mathcal{N}\left(\mathbb{R}^{m}\right)$ for all $\left(\eta_{\varepsilon}\right)_{\varepsilon} \in \mathcal{N}\left(\mathbb{R}^{m}\right)$, we have $L_{\varepsilon}\left(\psi_{\varepsilon^{a}} * f_{\varepsilon}-f_{\varepsilon}\right) * \varphi_{\varepsilon^{t}} \in \mathcal{N}\left(\mathbb{R}^{m}\right)$ and, finally $\widetilde{H}_{\varepsilon}\left(f_{\varepsilon}\right)-L_{\varepsilon}\left(f_{\varepsilon}\right) \in \mathcal{N}\left(\mathbb{R}^{m}\right)$. Thus $L\left(\left[\left(f_{\varepsilon}\right)_{\varepsilon}\right]\right)=\widetilde{H}_{L}\left(\left[\left(f_{\varepsilon}\right)_{\varepsilon}\right]\right)$ as expected. Due to 12 , we obtain that $\widetilde{H}_{L} \in \mathcal{G}^{\mathcal{L}_{2(a b+c)}}\left(\mathbb{R}^{m+n}\right)$ ending the proof.

\subsection{Proof of Proposition 3.9}

Assertion $(i)$. For a fixed $\varepsilon \in(0,1], L_{\varepsilon}$ is obtained by composition of the continuous maps $\Lambda: \mathcal{S}\left(\mathbb{R}^{n}\right) \mapsto \mathcal{S}^{\prime}\left(\mathbb{R}^{m}\right)$ and

$$
\mathcal{S}^{\prime}\left(\mathbb{R}^{m}\right) \rightarrow \mathcal{O}_{M}\left(\mathbb{R}^{n}\right), \quad T \mapsto T * \varphi_{\varepsilon} .
$$

Thus $L_{\varepsilon}$ is continuous. We have now to show that the net $\left(L_{\varepsilon}\right)_{\varepsilon} \in \mathcal{L}\left(\mathcal{S}\left(\mathbb{R}^{n}\right), \mathcal{O}_{M}\left(\mathbb{R}^{m}\right)\right)^{(0,1]}$ is strongly moderate. We have

$$
\begin{aligned}
\forall f \in \mathcal{S}\left(\mathbb{R}^{n}\right), \forall x \in \mathbb{R}^{m}, \forall \alpha \in \mathbb{N}^{m}, \partial^{\alpha}\left(L_{\varepsilon}(f)\right)(x) & =\left(\Lambda(f) * \partial^{\alpha} \varphi_{\varepsilon}\right)(x) \\
& =\left\langle\Lambda(f),\left\{y \mapsto \partial^{\alpha} \varphi_{\varepsilon}(x-y)\right\}\right\rangle .
\end{aligned}
$$

The map

$$
\Theta: \mathcal{S}\left(\mathbb{R}^{n}\right) \times \mathcal{S}\left(\mathbb{R}^{m}\right), \quad(f, \varphi) \rightarrow\langle\Lambda(f), \varphi\rangle,
$$

is a bilinear map, separately continuous since $\Lambda$ is continuous. As $\mathcal{S}\left(\mathbb{R}^{n}\right)$ and $\mathcal{S}\left(\mathbb{R}^{m}\right)$ are Fréchet spaces, $\Theta$ is globally continuous. There exist $C_{1}>0,\left(q_{1}, l_{1}, q_{2}, l_{2}\right) \in \mathbb{N}^{4}$, such that

$$
\forall(f, \varphi) \in \mathcal{S}\left(\mathbb{R}^{n}\right) \times \mathcal{S}\left(\mathbb{R}^{m}\right), \quad|\langle\Lambda(f), \varphi\rangle| \leq C_{1} \mu_{q_{1}, l_{1}}(f) \mu_{q_{2}, l_{2}}(\varphi) .
$$

In particular, for any $l \in \mathbb{N}$ and $\alpha \in \mathbb{N}^{m}$ with $|\alpha| \leq l$, we have

$$
\forall x \in \mathbb{R}^{m}, \quad\left|\left\langle\Lambda(f), \partial^{\alpha} \varphi_{\varepsilon}(x-\cdot)\right\rangle\right| \leq C_{1} \mu_{q_{1}, l_{1}}(f) \mu_{q_{2}, l_{2}}\left(\partial^{\alpha} \varphi_{\varepsilon}(x-\cdot)\right),
$$

with

$$
\begin{aligned}
\forall x \in \mathbb{R}^{m}, \quad \mu_{q_{2}, l_{2}}\left(\partial^{\alpha} \varphi_{\varepsilon}(x-\cdot)\right) & =\sup _{\xi \in \mathbb{R}^{m},|\beta| \leq l_{2}}(1+|\xi|)^{q_{2}}\left|\partial^{\alpha+\beta} \varphi_{\varepsilon}(x-\xi)\right| \\
& =\sup _{\xi \in \mathbb{R}^{m},|\beta| \leq l_{2}}(1+|x-\xi|)^{q_{2}}\left|\partial^{\alpha+\beta} \varphi_{\varepsilon}(\xi)\right| \\
& \leq(1+|x|)^{q_{2}} \mu_{q_{2}, l_{2}+l}\left|\varphi_{\varepsilon}\right| .
\end{aligned}
$$

Using the definition of $\left(\varphi_{\varepsilon}\right)_{\varepsilon}$, we get $C_{2}>0$ such that $\mu_{q_{2}, l_{2}+l}\left|\varphi_{\varepsilon}\right| \leq C_{2} \varepsilon^{-\left(m+l_{2}+l\right)}$, for $\varepsilon$ small enough. Thus, there exists $C>0$ such that, for $\varepsilon$ small enough,

$$
\forall x \in \mathbb{R}^{m}, \quad\left|\partial^{\alpha}\left(L_{\varepsilon}(f)\right)(x)\right|=\left|\left\langle\Lambda(f), \partial^{\alpha} \varphi_{\varepsilon}(x-\cdot)\right\rangle\right| \leq C(1+|x|)^{q_{2}} \mu_{q_{1}, l_{1}}(f) \varepsilon^{-\left(m+l_{2}+l\right)} .
$$

Finally

$$
\mu_{-q_{2}, l}\left(L_{\varepsilon}(f)\right) \leq C \varepsilon^{-\left(m+l_{2}+l\right)} \mu_{q_{1}, l_{1}}(f) .
$$

The sequence $r(\cdot)=\left\{l \mapsto\left(m+l_{2}+l\right)\right\}$ satisfies $\lim _{l \rightarrow+\infty}(r(l) / l)=1$. Recalling that $l_{1}$ does not depend on $l$, we obtain our claim.

Assertion (ii). Consider the kernel $H_{L_{\Lambda}}$ associated to $\left(L_{\varepsilon}(f)\right)_{\varepsilon}$ by Theorem 3.7. As $\sigma_{\mathcal{S}}\left(\mathcal{S}\left(\mathbb{R}^{m}\right)\right) \subset \mathcal{G}_{S}^{\mathcal{L}_{0}}\left(\mathbb{R}^{m}\right)$, we have $L_{\Lambda}(f)=\left[\left(L_{\varepsilon}(f)\right)_{\varepsilon}\right]_{\tau}=\widetilde{H}_{L_{\Lambda}}(f)$ by relation 10 . On the other hand, $\left[\left(L_{\varepsilon}(f)\right)_{\varepsilon}\right]_{\tau}=\left[\left(\Lambda(f) * \varphi_{\varepsilon}\right)_{\varepsilon}\right]_{\tau}=\iota_{\tau}(\Lambda(f))$ by definition of $\left(L_{\varepsilon}\right)_{\varepsilon}$. This proves our assertion, the commutativity of diagram (11) and ends the proof of Proposition 3.9 . 


\section{References}

[1] A. B. Antonevich and Ya. V. Radyno, On a general method for constructing algebras of generalized functions, Soviet Math. Dokl. 43 (1992), 680-684.

[2] S. Bernard, J.-F. Colombeau and A. Delcroix, Generalized integral operators and applications, Math. Proc. Cambridge Philos. Soc. 141 (2006), 521-546.

[3] J.-F. Colombeau, New Generalized Functions and Multiplication of Distributions, NorthHolland, Amsterdam, 1984.

[4] J.-F. Colombeau, Elementary Introduction to New Generalized Functions, North-Holland, Amsterdam, 1985.

[5] A. Delcroix, Generalized integral operators and Schwartz kernel theorem, J. Math. Anal. Appl. 306 (2005), 481-501.

[6] A. Delcroix, Regular rapidly decreasing nonlinear generalized functions. Application to microlocal regularity, J. Math. Anal. Appl. 327 (2007), 564-584.

[7] A. Delcroix, Kernel theorems in spaces of tempered generalized functions, Math. Proc. Camb. Philos. Soc. 142 (2007), 557-572.

[8] C. Garetto, Pseudo-differential operators in algebras of generalized functions and global hypoellipticity, Acta Appl. Math. 80 (2004), 123-174.

[9] C. Garetto, Topological structures in Colombeau algebras: investigation of the duals of $\mathcal{G}_{C}(\Omega), \mathcal{G}(\Omega)$, Monatsh. Math. 146 (2005), 203-226.

[10] C. Garetto, T. Gramchev and M. Oberguggenberger, Pseudo-differential operators and regularity theory, Electron. J. Diff. Eqns. 116 (2005), 1-43.

[11] M. Grosser, M. Kunzinger, M. Oberguggenberger and R. Steinbauer, Geometric Theory of Generalized Functions with Applications to General Relativity, Kluwer Academic Press, Dordrecht, 2001.

[12] L. Hörmander, The Analysis of Linear Partial Differential Operators I, Distribution Theory and Fourier Analysis, Grundlehren der mathematischen Wissenschaften 256, Springer Verlag, Berlin, 2nd edition, 1990.

[13] M. Nedeljkov, S. Pilipović and D. Scarpalézos, The Linear Theory of Colombeau Generalized Functions, Pitman Research Notes in Mathematics Series 385, Longman, Harlow, 1998.

[14] M. Oberguggenberger, Multiplication of Distributions and Applications to Partial Differential Equations, Longman Scientific \& Technical, Harlow, 1992.

[15] Ya. V. Radyno, Ngo Fu Tkhan, S. Ramadan, The Fourier transform in an algebra of new generalized functions, Russian Acad. Sci. Dokl. Math. 46 (1992), 414-417.

[16] D. Scarpalézos, Colombeau's generalized functions: Topological structures; Microlocal properties. A simplified point of view, preprint, Université de Paris 7, 1993.

[17] D. Scarpalézos, Colombeau's generalized functions: Topological structures; Microlocal properties. A simplified point of view. Part I, Bull. Cl. Sci. Math. Nat. Sci. Math. 25 (2000), 89-114.

[18] D. Scarpalézos, Colombeau's generalized functions: Topological structures; Microlocal properties. A simplified point of view. Part II, Publ. Inst. Math. (Beograd) (N.S.) 76(90) (2004), 111-125.

[19] L. Schwartz, Théorie des Distributions, Hermann, Paris, 3rd print., 1965.

[20] F. Treves, Topological Vector Spaces, Distributions and Kernels, Academic Press, New York, 1967.

[21] V. Valmorin, Schwartz kernel theorem in Colombeau algebras, this volume, 285-296. 
